\title{
The integrin expression profile modulates orientation and dynamics of force transmission at cell-matrix adhesions
}

\author{
Hayri E. Balcioglu ${ }^{1, *}$, Hedde van Hoorn ${ }^{2}$, Dominique M. Donato ${ }^{2}$, Thomas Schmidt ${ }^{2}$ and Erik H. J. Danen ${ }^{1, *}$
}

\begin{abstract}
Integrin adhesion receptors connect the extracellular matrix (ECM) to the cytoskeleton and serve as bidirectional mechanotransducers. During development, angiogenesis, wound healing and cancer progression, the relative abundance of fibronectin receptors, including integrins $\alpha 5 \beta 1$ and $\alpha v \beta 3$, changes, thus altering the integrin composition of cell-matrix adhesions. Here, we show that enhanced $\alpha \mathrm{v} \beta 3$ expression can fully compensate for loss of $\alpha 5 \beta 1$ and other $\beta 1$ integrins to support outside-in and inside-out force transmission. $\alpha 5 \beta 1$ and $\alpha v \beta 3$ each mediate actin cytoskeletal remodeling in response to stiffening or cyclic stretching of the ECM. Likewise, $\alpha 5 \beta 1$ and $\alpha v \beta 3$ support cellular traction forces of comparable magnitudes and similarly increase these forces in response to ECM stiffening. However, cells using $\alpha v \beta 3$ respond to lower stiffness ranges, reorganize their actin cytoskeleton more substantially in response to stretch, and show more randomly oriented traction forces. Centripetal traction force orientation requires long stress fibers that are formed through the action of Rho kinase (ROCK) and myosin II, and that are supported by $\alpha 5 \beta 1$. Thus, altering the relative abundance of fibronectin-binding integrins in cell-matrix adhesions affects the spatiotemporal organization of force transmission.
\end{abstract}

KEY WORDS: Cell-matrix adhesion, $\beta 1$ integrin, $\beta 3$ integrin, Mechanotransduction, Actin cytoskeleton, Fibronectin, Rho kinase, ROCK, Traction force

\section{INTRODUCTION}

Cells sense the mechanical properties of their surrounding environment and activate intracellular signaling cascades generating an elaborate response that plays a role in cell survival, proliferation, differentiation and migration (Hoffman et al., 2011). Cell-matrix adhesions are dynamic force-responsive protein complexes that couple the extracellular matrix (ECM) to the cytoskeleton (Schiller and Fässler, 2013). Within these adhesions, integrin $\alpha-\beta$ heterodimeric transmembrane receptors bind ECM proteins with their globular head domains and connect to the cytoskeleton through multi-protein interactions at their cytoplasmic tails (Hynes, 2002). Integrins transmit forces in a bidirectional manner: extracellular forces applied to the head domains enhance integrin activity and clustering, and trigger

${ }^{1}$ Division of Toxicology, Leiden Academic Center for Drug Research, Leiden University, 2333 CC Leiden, the Netherlands. ${ }^{2}$ Physics of Life Processes,

Kamerlingh Onnes-Huygens Laboratory, Leiden University, 2333 CC Leiden, the Netherlands.

*Authors for correspondence (h.e.balcioglu@lacdr.leidenuniv.nl;

e.danen@lacdr.leidenuniv.nl)

Received 22 May 2014; Accepted 2 February 2015 cell-matrix adhesion growth and cytoskeletal reorganization. Vice versa, actomyosin-mediated contractile forces cause strengthening of integrin-ECM binding (Choquet et al., 1997; Friedland et al., 2009; Guilluy et al., 2011; Roca-Cusachs et al., 2012).

Cell-matrix adhesions formed on fibronectin contain a mixture of different integrins, including $\alpha 5 \beta 1$ and $\alpha \mathrm{v} \beta 3$. When cells are stimulated to move or proliferate during development, angiogenesis or tissue regeneration, shifts in the relative abundance of these fibronectin-binding integrins occur (Bouvard et al., 2013; Wolfenson et al., 2013). Likewise, alterations in the abundance of $\alpha 5 \beta 1$ or $\alpha v \beta 3$ take place during cancer progression (Desgrosellier and Cheresh, 2010). Such changes will alter the integrin composition of cell-matrix adhesions, and we and others have previously shown that this affects cytoskeletal organization, activity of Rho GTPases and migratory behavior (Danen et al., 2002; Danen et al., 2005; Miao et al., 2002).

Using mouse embryonic stem cell (ESC)-derived fibroblastic cells (GD25) and mouse-embryo-derived neuroepithelial cells (GE11) lacking the common $\beta 1$ subunit, we have shown that reexpression of $\beta 1$ (but not increased expression of $\beta 3$ supporting a similar level of adhesion to fibronectin) stimulates contractility mediated by RhoA and Rho kinase (ROCK, for which there are two isoforms ROCK1 and ROCK2) and more-random migration (Danen et al., 2002; Danen et al., 2005). Likewise, White et al. have shown that prevention of $\alpha v \beta 3$ recycling in NIH3T3 cells, thereby causing enhanced surface abundance of $\alpha 5 \beta 1$, stimulates ROCK-mediated contractility and random movement (White et al., 2007). Conversely, Miao et al. have demonstrated that expression of $\beta 3$ integrins (but not increased expression of $\beta 1$ integrins) in $\mathrm{CHO}$ cells that lack $\beta 3$ causes enhanced RhoA and ROCK activity (Miao et al., 2002). This suggested that the total amount of fibronectin-binding integrins is more relevant or that expression of both $\beta 1$ and $\beta 3$ integrins is needed for effective Rho-ROCKmediated contractility. However, we have shown that $\beta 3$-knockout MEFs have no defect in RhoA-ROCK-mediated contractility and ectopic expression of $\beta 3$ integrins does not further stimulate this pathway (whereas increased expression of $\beta 1$ integrins does) (Huveneers et al., 2008). Moreover, like expression of $\beta 1$ integrins in $\beta 1$-null cells; expression of $\alpha 5$ in $\alpha 5$-null mouse ESC-derived fibroblastic cells also stimulates RhoA-ROCKmediated contractility (Huveneers et al., 2008).

It has subsequently become clear that different integrins can mediate distinct signaling routes that support distinct aspects of mechanotransduction. Experiments using MEFs in which ligandcoated beads were used to pull on small integrin clusters have shown that $\alpha 5 \beta 1$ mediates adhesion strength whereas $\alpha \mathrm{v} \beta 3$ mediates cytoskeletal stiffening (Roca-Cusachs et al., 2009). A recent study using pan-integrin knockout kidney fibroblasts reconstituted with $\alpha \mathrm{v}, \beta 1$ or both subunits, resulting in equimolar 
surface levels of $\alpha 5 \beta 1$ and/or $\alpha \mathrm{v} \beta 3$ and $\alpha \mathrm{v} \beta 5$ has provided further insight: $\alpha 5 \beta 1$-mediated adhesion indeed stimulates RhoA-ROCK signaling to activate myosin II but $\alpha \mathrm{v}$ integrins are required to support RhoA-mDia-mediated actin polymerization and these processes cooperate to regulate contractility (Schiller et al., 2013). Thus, the expression levels of $\alpha 5 \beta 1, \alpha \mathrm{v} \beta 3$, as well as other $\alpha \mathrm{v}-$ integrins participating in fibronectin-binding (e.g. $\alpha \mathrm{v} \beta 1$ and $\alpha \mathrm{v} \beta 6$ ) in combination with the distinct signaling networks of integrinassociated proteins present in embryonic or ESC-derived epithelial or fibroblastic cells, kidney cells, or CHO cells used in the abovementioned studies ultimately determine the outcome of changes in the fibronectin-receptor repertoire for RhoA-mediated signaling and cytoarchitecture.

In this study, we asked to what extent a shift from $\alpha 5 \beta 1$ to more $\alpha \mathrm{v} \beta 3$ expression, as often seen during angiogenesis, wound healing or cancer progression, affects mechanotransduction. We used two independent cell systems in which adhesion to fibronectin is mediated mainly by $\alpha 5 \beta 1$ or by $\alpha v \beta 3$ integrins resulting in comparable adhesion efficiency and compared the ability of such cells to (1) sense and respond to extracellular forces (outside-in signaling), and (2) exert forces onto the ECM (inside-out signaling).

\section{RESULTS}

Cells adhering through $\alpha v \beta 3$ show a more substantial cytoskeletal reorganization in response to cyclic stretch as compared to cells using $\alpha 5 \beta 1$

To compare responses to extracellular forces we made use of GE $\beta 1$ and GE $\beta 3$ cells. These cells are derived from $\beta 1$ integrin chimeric mouse embryos lacking the common $\beta 1$ subunit and were engineered to express human $\beta 1$ or $\beta 3$ subunits. Fluorescenceactivated cell sorting (FACS) showed that ectopically expressed $\beta 1$ and $\beta 3$ led to high cell surface levels but these did not exceed endogenous levels observed in MDA-MB-435s human breast cancer cells (supplementary material Fig. S1C,D,G,I). GE $\beta 1$ and GE $\beta 3$ cells have been previously shown to support adhesion to fibronectin-coated glass substrates with the same efficiency through either $\alpha 5 \beta 1$ or $\alpha v \beta 3$, respectively (Danen et al., 2002). The cells were transduced with mCherry-LifeAct for actin imaging (supplementary material Fig. S1E,F,I,J) and plated on a fibronectin-coated poly(dimethyl)siloxane (PDMS) membrane and subjected to uniaxial cyclic stretch first at $10 \% 1 \mathrm{~Hz}$ for 2 hours, then at $20 \% 1 \mathrm{~Hz}$ for 1 hour (Fig. 1A). Incubation with integrin blocking antibodies confirmed that, like fibronectin-coated glass substrates, GE $\beta 1$ and GE $\beta 3$ adhered to fibronectin-coated PDMS substrates mainly through $\alpha 5 \beta 1$ and $\alpha \mathrm{v} \beta 3$, respectively (supplementary material Fig. S2H). Upon cyclic stretch, both GE $\beta 1$ and GE $\beta 3$ cells showed a gradual decrease in cell-spreading area with the two subsequent stretching regimes. The total actin filament length showed the same trend for GE $\beta 1$ cells, but for GE $\beta 3$ cells the total filament length already approached a minimum value at $10 \%$ stretch and showed only a slight additional decrease after a subsequent 20\% stretching (Fig. 1B-E).

PDMS membranes coated with fluorescent beads or stamped with patterned fluorescently labeled fibronectin were used to characterize the strain field over the membrane, the dynamic strain in the imaging field and to determine the angle of minimal strain (Fig. 1A; supplementary material Fig. S2A-D). GE $\beta 1$ cells oriented their F-actin towards the minimal strain direction $\left(\sim 60^{\circ}\right.$ to the macroscopic strain) following the $10 \%$ stretch regime, but this response was lost during the subsequent, second regime of $20 \%$ stretch (Fig. 1F,H). GE $\beta 3$ cells subjected to the first stretch regime showed a more prominent actin filament orientation towards the minimal strain direction and this response was maintained during the $20 \%$ stretch regime (Fig. $1 \mathrm{G}, \mathrm{H}$ ).

These findings indicate that cells adhering mainly through $\alpha 5 \beta 1$ or $\alpha v \beta 3$ integrins can both sense cyclic ECM strain and trigger actin cytoskeleton remodeling. However, high expression of $\alpha v \beta 3$ allows cells to more effectively reorient their cytoskeleton in the direction of minimal strain and maintain this orientation at high strain rates.

\section{Cells expressing $\alpha 5 \beta 1$ or $\alpha v \beta 3$ each support cell spreading in response to substrate stiffening}

Next, we seeded GE $\beta 1$ and GE $\beta 3$ cells onto fibronectincrosslinked polyacrylamide (PAA) gels with shear moduli varying between $760 \mathrm{~Pa}$ and $13.4 \mathrm{kPa}$ (supplementary material Fig. S2F,G). Incubation with integrin-blocking antibodies confirmed that, like fibronectin-coated glass and PDMS substrates, GE $\beta 1$ and GE $\beta 3$ adhered to fibronectin-crosslinked PAA substrates mainly through $\alpha 5 \beta 1$ and $\alpha \mathrm{v} \beta 3$, respectively (supplementary material Fig. S2I). Both cell types showed a gradual increase in cell spreading area with increasing stiffness (Fig. 2A,C). Similar findings were obtained using the GD25 cell line derived from $\beta 1$-null ESCs where expression of human $\beta 1$ or $\beta 3$ subunits supports adhesion to fibronectin with the same efficiency through $\alpha 5 \beta 1$ or $\alpha v \beta 3$, respectively (Danen et al., 2002) and had comparable surface expression levels of these integrins to those in MDA-MB-435s cells (supplementary material Fig. S1A,B,H,I). It should be noted that, for GD cells, lower stiffness ranges were used as compared to those used for the GE cell lines given that full cell spreading is already observed on softer substrates for this cell type. Again, cell-spreading area increased with increasing stiffness over the range of stiffnesses tested for cells adhering through either of these integrins (Fig. 2B,D). Non-linear fitting using a cumulative Gaussian distribution (supplementary material Fig. S3A) showed that despite having significantly different response curves (supplementary material Fig. S3B-D) the estimated half response stiffness $\left(E_{1 / 2}\right)$ was not integrin specific (Fig. 2A,B).

\section{Cells adhering through $\alpha v \beta 3$ form cell-matrix adhesions at lower substrate stiffness compared to cells adhering through $\alpha 5 \beta 1$}

Similar to cellular area, the number of peripheral cell-matrix adhesions increased with increasing stiffness for all cell lines. For GE $\beta 3$ and GD $\beta 3$ cells the number of peripheral cell-matrix adhesions reached its maximum at intermediate stiffness, with an elastic modulus of 9.4 and $5.47 \mathrm{kPa}$, respectively (Fig. 3A,B,D; supplementary material Fig. S3J). By contrast, the number of cell-matrix adhesions in GE $\beta 1$ and GD $\beta 1$ cells showed a more gradual increase over the entire range of stiffnesses tested (Fig. 3A-C; supplementary material Fig. S3I). The half-response stiffness $\left(E_{1 / 2}\right)$ was also significantly lower for cells using $\alpha v \beta 3$, as compared to that for cells using $\alpha 5 \beta 1$ (Fig. 3A,B; supplementary material Fig. S3B,E,F). The average cell-matrix adhesion size did not show the same gradual response to rigidity: once adhesions were formed, they reached similar sizes irrespective of the ECM stiffness (Fig. 3C,D; supplementary material Fig. S3G-J).

Taken together, these findings demonstrate that cells expressing $\alpha 5 \beta 1$ and $\alpha \mathrm{v} \beta 3$ can each sense - and respond to variations in substrate stiffness, but that $\alpha \mathrm{v} \beta 3$ supports cellmatrix adhesion formation more readily at a lower stiffness. 

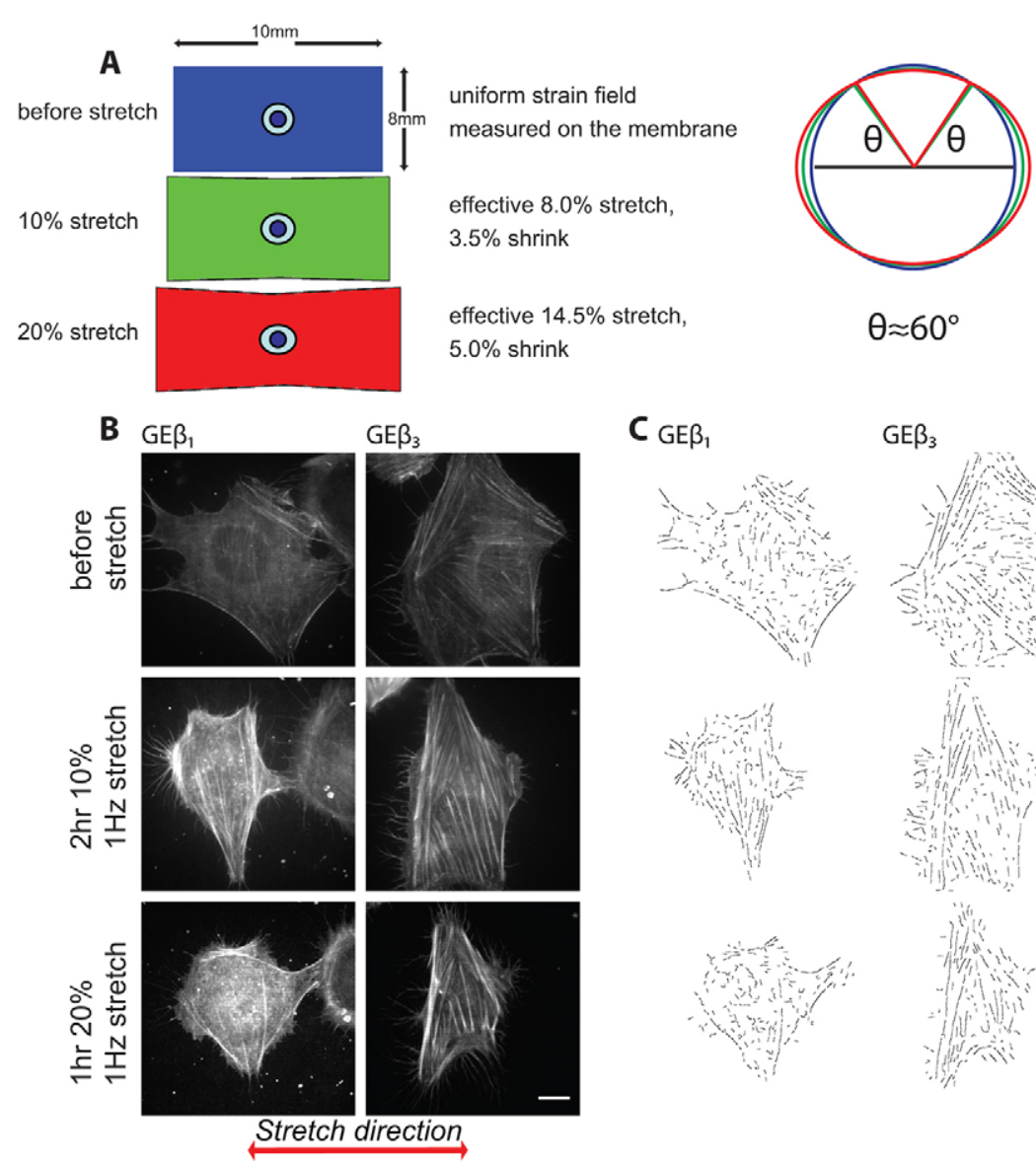

\section{$C_{G E \beta_{1}}$}

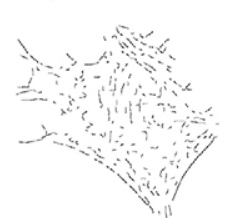

$\mathrm{GE} \beta_{3}$
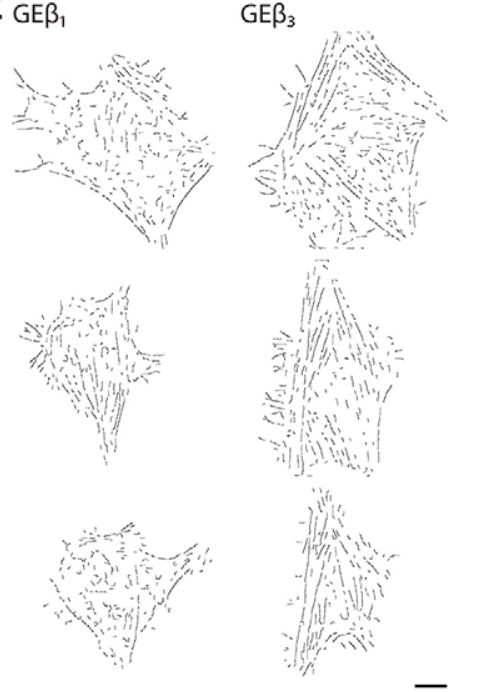

Fig. 1. Cells expressing $\alpha \mathrm{v} \beta 3$ integrins more effectively reorganize their cytoskeleton upon cyclic stretch. (A) Stretch regimes used during the experiment. Driving the piezo controllers with $10 \%$ or $20 \%$ displacement resulted in $8 \%$ or $14.5 \%$ stretch on the PDMS membrane in the direction of the displacement, and $3.5 \%$ or $5 \%$ shrink in the perpendicular direction, respectively. This resulted in a minimal strain angle of $\sim 60^{\circ}$. (B) mCherry-LifeActtransduced GE $\beta 1$ and GE $\beta 3$ cells respond to twostep cyclic stretch. (C) Characterization of actin organization for the cells shown in B.

$(D, E)$ Quantification of cellular spread area (D) and total actin filament length (E) for the cells indicated. Mean $\pm 95 \%$ clearance level of $>75$ cells from three independent experiments. $(F, G)$ Angular organization of actin filaments averaged over all GE $\beta 1$ (F) or GE $\beta 3$ (G) cells measured. Measured angle of orientation of actin filaments is relative to stretch direction. The gray bar indicates region of minimal strain. $(\mathrm{H})$ Average actin filament orientation per cell. Mean $\pm 95 \%$ clearance level of $>75$ cells from three independent experiments. NS, $P>0.05$; ${ }^{*} P<0.05$; ${ }^{*} P<0.005$;

${ }^{* * *} P<0.0005$ according to Mann-Whitney test. Scale bars: $10 \mu \mathrm{m}$.
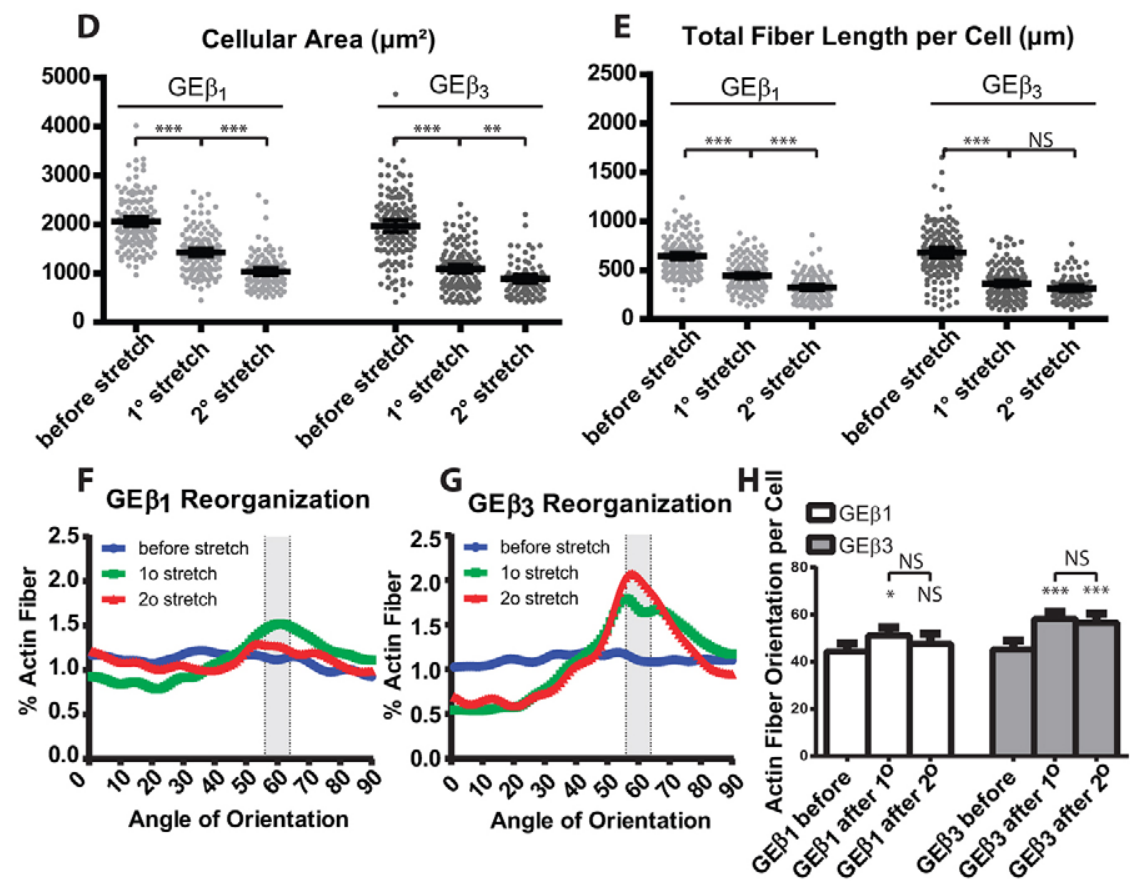

\section{Cells adhering through $\alpha 5 \beta 1$ or $\alpha v \beta 3$ each mediate traction forces that are regulated in response to altered substrate rigidity}

Having examined the consequences of expression of either $\alpha 5 \beta 1$ or $\alpha v \beta 3$ for outside-in cellular responses to extracellular forces, we next investigated whether these integrins differed in their ability to mediate inside-out cellular traction forces onto the ECM. Therefore, mCherry-LifeAct-expressing GE $\beta 1$ and GE $\beta 3$ cells were seeded on fibronectin-stamped PDMS micropillars of $6.9 \mu \mathrm{m}$ height (bending stiffness of $16 \mathrm{nN} / \mu \mathrm{m}$ ). Cell spreading on these micropillars, as well as the mean force per pillar, was similar for both cell lines (Fig. 4A-C). This indicated that $\beta 1$ 


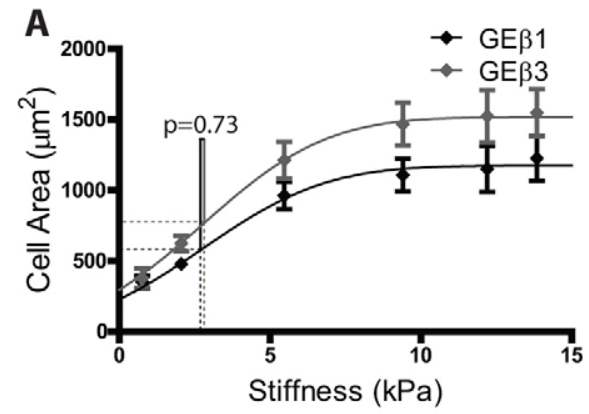

C

$\mathrm{GE}_{1}{ }_{760 \mathrm{~Pa}}$

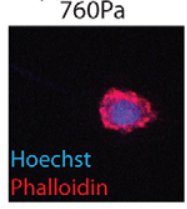

$\mathrm{GE} \beta_{3}$

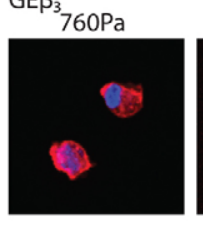

$9.4 \mathrm{kPa}$

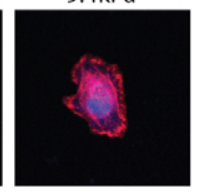

$9.4 \mathrm{kPa}$

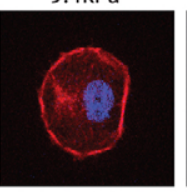

B

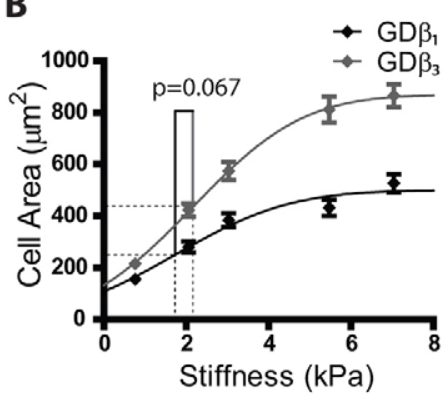

D

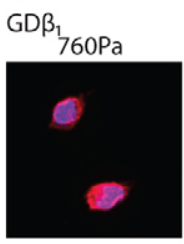

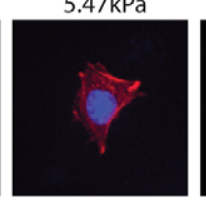

$5.47 \mathrm{kPa}$

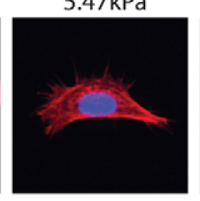

$\mathrm{GD} \beta_{3}$

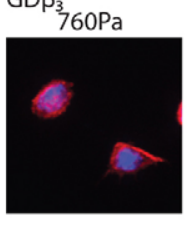

$5.47 \mathrm{kPa}$

glass

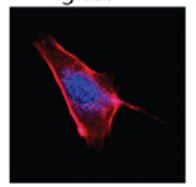

glass

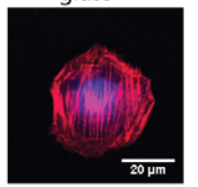

glass

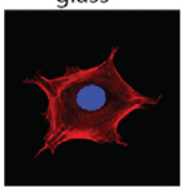

glass

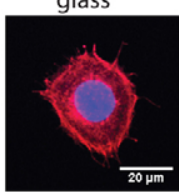

Fig. 2. Cells respond to increased substrate stiffness by increased spreading irrespective of the integrin engaged. $(A, B)$ Quantification of cellular spread area of GE $\beta 1$ and GE $\beta 3$ cells (A) or GD $\beta 1$ and GD $\beta 3$ cells (B) over measured rigidities and cumulative Gaussian distribution model fitted. Mean $\pm 95 \%$ clearance level is shown. $>100$ cells were measured from three independent experiments (except at $760 \mathrm{~Pa}$ for GE $\beta 1$ and GE $\beta 3$ cells where more than 60 cells were measured from a single experiment). $P$-values were calculated by comparing the halfway points of the cumulative Gaussian fits with an F-test. (C,D) Representative images of cells described in A and B. Scale bars: $20 \mu \mathrm{m}$.

integrins were not required for the generation of traction forces in cells where $\alpha v \beta 3$ levels are sufficiently high to compensate for adhesion, despite earlier reports pointing to a crucial role for $\beta 1$ integrins (Roca-Cusachs et al., 2009; Schiller et al., 2013). To address whether expression of $\beta 1$ integrins might further increase traction forces in GE $\beta 3$ cells, we plated GE $\beta 1+\beta 3$ and GE $\beta 3+\beta 1$ cells on $6.9 \mu \mathrm{m}$ fibronectin-stamped micropillars. However, comparable cell spreading and forces were measured for these cells as observed for GE $\beta 1$ and GE $\beta 3$ cells (Fig. 4A,B). Taken together, these findings indicate that traction forces can be generated irrespective of the type of fibronectin-binding integrin expressed.

We next analyzed the ability of these cells to increase traction forces in response to increased substrate stiffness. Plating cells on shorter pillars ( $4.1 \mu \mathrm{m}$ height; bending stiffness of $66 \mathrm{nN} / \mu \mathrm{m})$ led to increased cell spreading and to $\sim$ twofold increase in traction forces, irrespective of the integrin used (Fig. 4D-F). The increase in traction force was $\sim$ threefold for GE $\beta 1+\beta 3$ and GE $\beta 3+\beta 1$ cells indicating that the total amount of fibronectin-binding integrins might determine the magnitude of the response (Fig. 4E). The twofold increase in response to substrate stiffening was maintained for GE $\beta 1$ and GE $\beta 3$ cells in post-fixation samples and GD $\beta 1$ and GD $\beta 3$ cells each showed a similar response although the magnitude of the response to stiffening was lower for GD $\beta 3$ cells (supplementary material Fig. S4A-C). In addition, a similar, albeit somewhat stronger increase in traction forces upon seeding on shorter pillars was observed for NIH3T3 cells that bind to fibronectin through both $\alpha 5 \beta 1$ and $\alpha v \beta 3$ (Woods et al., 2001) (supplementary material Fig. S4A,B). Finally, having established that the initial adhesion to fibronectin-coated PDMS involved $\alpha 5 \beta 1$ for GE $\beta 1$ cells and $\alpha v \beta 3$ for GE $\beta 3$ cells (supplementary material Fig S2H), we analyzed the potential role of $\alpha \mathrm{v}$ integrins in the traction forces exerted by these cells. As expected, $\alpha \mathrm{v}$-integrinblocking antibodies decreased force application by GE $\beta 3$ cells, but they did not affect traction forces in GE $\beta 1$ cells, indicating that $\alpha 5 \beta 1$ is the major integrin responsible for force application on fibronectin in GE $\beta 1$ cells (supplementary material Fig. S4D-F).

These results indicate that cells are able to exert traction forces and respond to increased ECM stiffness by enhanced force application, irrespective of the type of fibronectin receptor engaged. Notably, the approximated shear modulus of 3.87 and $15.7 \mathrm{kPa}$ of these long and short pillars (see Materials and Methods section), was within the outside-in-sensing regimes tested using PAA substrates (Figs 2, 3).

\section{Cells adhering through $\alpha 5 \beta 1$ preferentially support centripetal force application and long actin filaments in an actomyosin contractility-dependent manner}

Cells expressing $\alpha 5 \beta 1$ or $\alpha v \beta 3$ show distinct organizations of the actin cytoskeleton and cell-matrix adhesions, with $\alpha 5 \beta 1$ supporting predominantly concave cortical actin structures (Danen et al., 2002; Danen et al., 2005) (Fig. 2C,D). We hypothesized that the morphology supported by $\alpha 5 \beta 1$ was related to more centripetally oriented forces (i.e. forces directed towards the cell center) exerted at cell-matrix adhesions. In order to investigate this, we analyzed the centripetally oriented force fraction compared to the total force. Live measurement of traction forces on $6.9 \mu \mathrm{m}$ and $4.1 \mu \mathrm{m}$ pillars showed that the centripetal force fraction in GE $\beta 1$ cells was slightly, but significantly, higher than that observed in GE $\beta 3$ cells (Fig. 5A, left panel). The centripetal force fraction in GE $\beta 1+\beta 3$ and GE $\beta 3+\beta 1$ cells was comparable to that in GE $\beta 1$ cells. The higher centripetal force fraction in $\beta 1$-expressing cells was also observed in post-fixation samples of GD $\beta 1$, GD $\beta 3$, GE $\beta 1$ and GE $\beta 3$ cells on $4.1 \mu \mathrm{m}$ pillars (Fig. 5A, middle panel).

We measured cortical actin filament lengths in GE $\beta 1$ and GE $\beta 3$ cells on $4.1 \mu \mathrm{m}$ pillars and noticed that higher centripetal force orientation in GE $\beta 1$ cells correlated with longer average cortical actin filament length (Fig. 5B,C). This suggests that the longer actin filaments in $\alpha 5 \beta 1$-expressing cells, rather than 

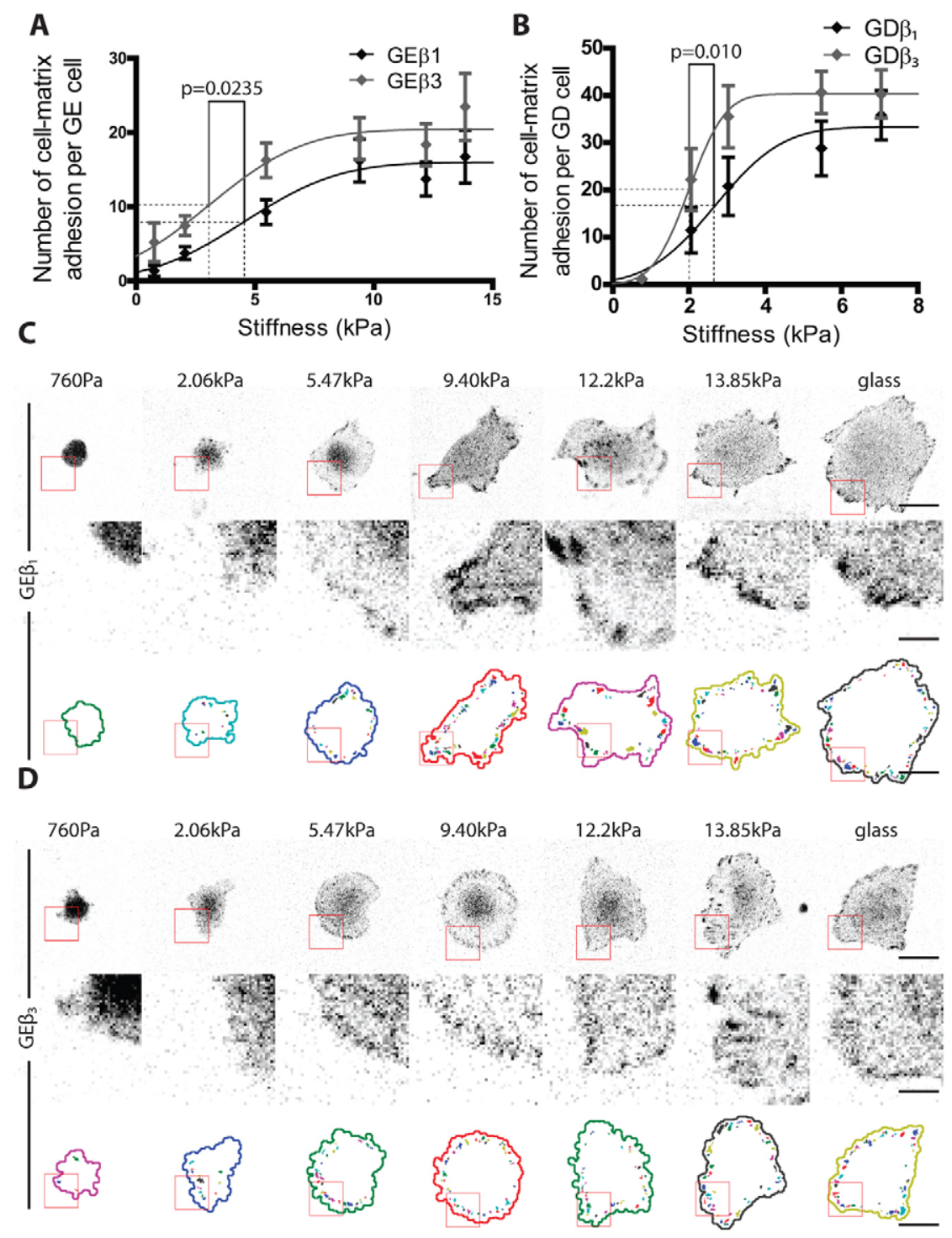

Fig. 3. The number of cell-matrix adhesions increases with increasing stiffness in an integrincontrolled manner. $(A, B)$ Quantification of number of peripheral cell-matrix adhesions of GE $\beta 1$ and GE $\beta 3$ cells $(A)$ or GD $\beta 1$ and GD $\beta 3$ cells $(B)$ over measured rigidities and fitted cumulative Gaussian distribution function. In all graphs, mean $\pm 95 \%$ clearance level is shown and at least 20 cells were measured over three independent experiments (except for $760 \mathrm{~Pa}$ for GE $\beta 1$ and GE $\beta 3$ cells where results of one experimental replica is shown). $P$-values were calculated by comparing the halfway points of the cumulative Gaussian fits $(A, B)$ with an F-test.

$(C, D)$ Representative images of paxillin (top), zoomed in region of the boxed area (middle) and adhesions detected by the automated analysis algorithm (bottom). Scale bar: $20 \mu \mathrm{m}$ (5 $\mu \mathrm{m}$ for magnified images). shorter actin cables in $\alpha v \beta 3$-expressing cells, support the centripetal orientation of forces. We and others have previously observed that $\alpha 5 \beta 1$ supports ROCK-mediated actomyosin contractility (Danen et al., 2002; Danen et al., 2005; Schiller et al., 2013; White et al., 2007) and we tested whether ROCK signaling was involved in the centripetal orientation of applied forces. Indeed, inhibition of ROCK or withdrawal of serum [containing lysophosphatidic acid, a known stimulator of RhoROCK signaling (Mills and Moolenaar, 2003)] reduced the centripetal orientation of force (Fig. 5A, right panel). These treatments also, though less effectively, reduced the average cortical actin filament length in GE $\beta 1$ to the level observed for GE $\beta 3$ (Fig. 5B,D). Likewise, treatment of GE $\beta 1$ cells with the myosin II inhibitor blebbistatin or disruption of the actin cytoskeleton with latrunculin B left only short actin cables intact and abolished the centripetal force orientation (Fig. 5B,D).

Taken together, these data show that even though traction forces mediated by $\alpha 5 \beta 1$ and $\alpha v \beta 3$ (possibly supported by other fibronectin-binding $\alpha \mathrm{v}$ integrins) are similar in magnitude; orientation of these forces is differentially regulated. This difference is related to long-range cortical actomyosin fibers supported by ROCK and myosin II in the context of $\alpha 5 \beta 1$ versus shorter actin cables in the context of $\alpha v \beta 3$ (Fig. 6).

\section{DISCUSSION}

Cell-matrix adhesions couple the ECM to the F-actin network and are regions for force transmission, allowing cells to adapt to the mechanical properties of the environment and to exert forces needed to remodel their environment. Our findings demonstrate that cell matrix adhesions can function as bi-directional force transducers irrespective of whether they contain $\alpha 5 \beta 1$ (and very little $\alpha v \beta 3$ ) or $\alpha v \beta 3$ (in the absence of any $\beta 1$ integrins). It should be noted that a contribution of alternative $\alpha \mathrm{v}$ integrins, such as $\alpha v \beta 5, \alpha v \beta 6$ or $\alpha v \beta 8$, and, in the case of cells expressing $\beta 1$ integrins, also $\alpha \mathrm{v} \beta 1$ cannot be fully ruled out in our study. Integrins $\alpha 5 \beta 1$ and $\alpha v \beta 3$ have been shown to play distinct roles in adhesion strengthening and actin cytoskeletal stiffening in integrin clusters under force (Roca-Cusachs et al., 2009). Our findings show that this does not translate into reduced force application by cellmatrix adhesions in the absence of $\alpha 5 \beta 1$ or ineffective F-actin reorganization when $\alpha \mathrm{v} \beta 3$ expression is low provided there is compensation through enhanced expression of $\alpha \mathrm{v} \beta 3$ or $\alpha 5 \beta 1$, 
A

Cellular area $\left(\mu \mathrm{m}^{2}\right)$

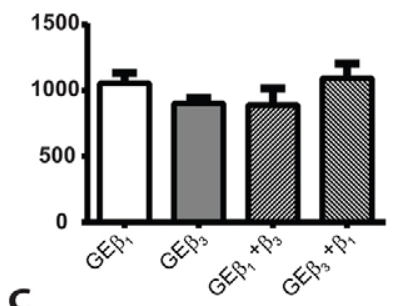

C

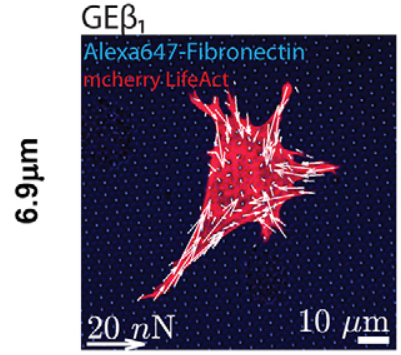

D

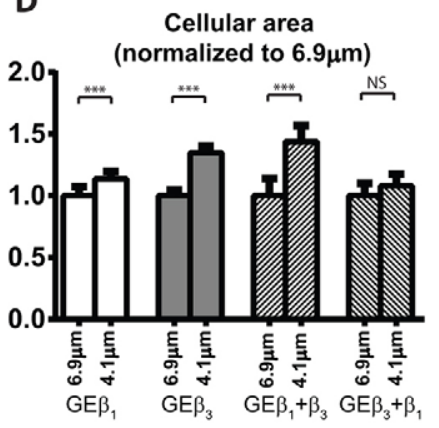

$\mathbf{F}$

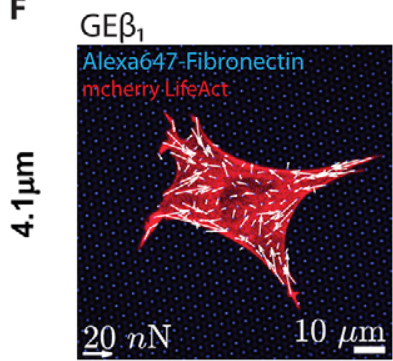

B

Force per pillar $(\mathrm{nN})$

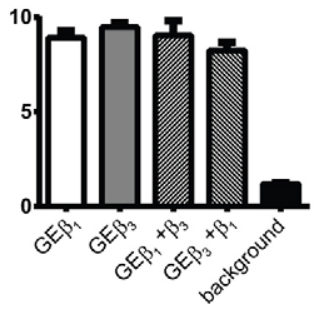

$\mathrm{GE} \beta_{3}$

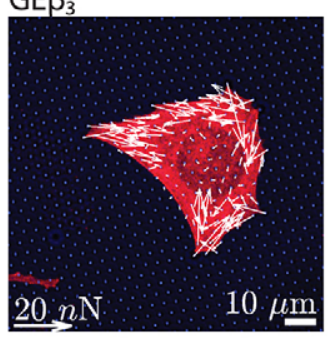

E

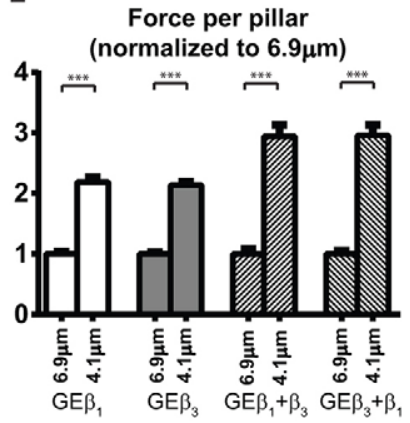

$\mathrm{GE} \beta_{3}$

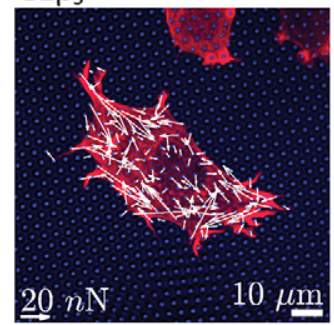

Fig. 4. Cellular traction force generation is similar for cells using $a 5 \beta 1$ or $\alpha \mathrm{v} \beta 3$ integrins. $(\mathrm{A}, \mathrm{B})$ Bar plots of cellular spread area and force per pillar of the indicated cell lines seeded on $6.9 \mu \mathrm{m}$ PDMS pillars. Background indicates forces measured in areas not covered by cells. (C) Representative images from $A, B$; white arrows indicate magnitude and direction of forces measured. Scale bar: $20 \mathrm{nN}$ and $10 \mu \mathrm{m}$. (D,E) Bar plots of cellular spread area and force per pillar of the indicated cell lines seeded on $4.1 \mu \mathrm{m}$ PDMS pillars, relative to measurements on $6.9 \mu \mathrm{m}$ pillars. In all graphs, the mean $\pm 95 \%$ clearance level is shown and at least 30 cells were measured over 3 independent experiments. (F) Representative images from D,E. White arrows and scale bars are as in $\mathrm{C}$.

respectively. Cells expressing either $\alpha 5 \beta 1$ or $\alpha \mathrm{v} \beta 3$ each respond to cyclic substrate stretching and each can sense variations in substrate stiffness and accordingly trigger cell spreading and cellmatrix adhesion formation. Likewise, both integrins allow cell matrix adhesions to apply traction forces onto the ECM and to respond to increased stiffness with enhanced force application.

Nevertheless, the manner in which force transduction is dynamically organized in cells expressing either of these integrins does differ. Our findings indicate that cells expressing $\alpha v \beta 3$ form cell matrix adhesions more effectively at lower substrate stiffnesses and more robustly reorganize their actin cytoskeleton to find the minimal strain in response to substrate stretching. It has been reported that substrate stretching triggers phosphoinositide 3-kinase (PI3K)-mediated $\alpha \mathrm{v} \beta 3$ activation, which in turn stimulates cellular responses including JNK activation (Katsumi et al., 2005). It will be of interest to explore whether such a mechanism underlies the effective cytoskeletal reorganization observed in cells expressing high levels of $\alpha v \beta 3$. The emergence of $\alpha v \beta 3$, which is frequently observed during active processes, such as angiogenesis or cancer invasion (Desgrosellier and Cheresh, 2010), might provide endothelial or tumor cells in these cases with enhanced flexibility to adapt their cytoarchitecture to ECM properties and activate cellular signaling in soft environments.

Our findings indicate that cells using $\alpha 5 \beta 1$ or $\alpha v \beta 3$ respond to substrate stiffening by cell spreading, cell matrix adhesion formation, and by applying more force to the substrate. It has been demonstrated that $\alpha \mathrm{V}$-integrins support coupling of RhoA activity to mDia, which drives actin polymerization (Schiller et al., 2013). Unlike that study, our experiments do not test such a role for fibronectin-binding $\alpha \mathrm{v}$-integrins in mechanotransduction; $\alpha \mathrm{V}$-integrins are expressed in all cell variants tested in our study $(\alpha v \beta 1$ and others in GE $\beta 1$ and GD $\beta 1 ; \alpha v \beta 3$ and others in GE $\beta 3$ and GD $\beta 3$ ). Unlike earlier reports (Roca-Cusachs et al., 2009; Schiller et al., 2013), we do not observe a marked deficiency in traction force induction by cells lacking $\beta 1$ integrins when $\alpha \mathrm{v} \beta 3$ is expressed at sufficient levels to fully rescue the adhesion defect. Notably, expression levels in our study are comparable to endogenous levels of $\beta 1$ or $\beta 3$ found in cancer cells. The role of fibronectin-binding integrins in traction force generation appears to differ for different cell types. Besides variations in the profile of $\alpha \mathrm{v}$ integrins for which the distinct roles in cytoskeletal organization are poorly understood, the integrin-associated signaling complex, including Rho GTPases and their upstream regulators and downstream effectors, might differ considerably in the variety of cell types used in different studies. This makes a direct comparison of different studies exploring integrin-mediated control of cytoskeletal organization and mechanotransduction difficult.

It has been reported that extracellular stimuli leading to activation of $\alpha 5 \beta 1$, but not those causing activation of $\alpha v \beta 3$, can trigger cell traction forces (Lin et al., 2013). In that study, the authors measured total force per cell, a parameter that is sensitive to effects on cell spreading area. Instead, here we determined force per pillar, which is independent of cell spreading area, and show that the induction of traction forces in response to extracellular stiffening can occur through both $\alpha 5 \beta 1$ and $\alpha v \beta 3$. The report from Lin et al. and our current study differ in the stimuli that are used (antibody-mediated integrin activation versus substrate stiffening through pillar shortening) and in the cell types that are tested, which might regulate force transmission differently. Our findings show that both integrins can be used by cells to sense alterations in the physical properties of the environment and to respond to such changes by modulation of traction forces exerted onto the ECM.

Rather than a role for $\alpha 5 \beta 1$ in force generation per se, which we show can be compensated for by enhanced expression of $\alpha v \beta 3$ in complete absence of $\beta 1$ integrins, we demonstrate that the orientation of forces is determined by the absence or presence of $\alpha 5 \beta 1$. This integrin allows cells to maintain contractile forces directed to the center of the cell and in its absence, forces become 
A

\section{Centripetal Force Percentage}

Live

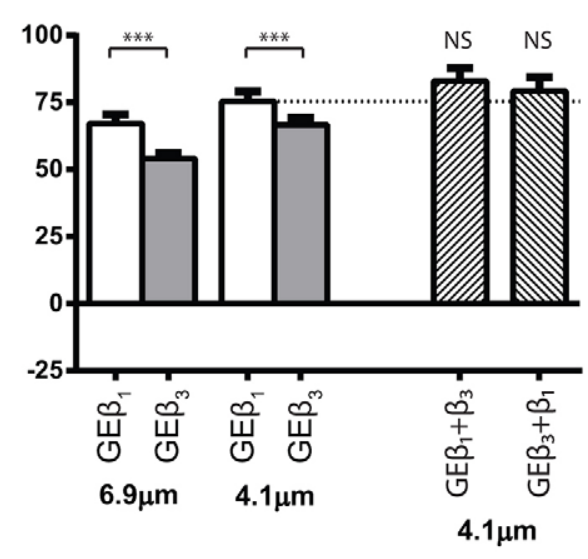

B

Average cortical actin fiber length $(\mu \mathrm{m})$

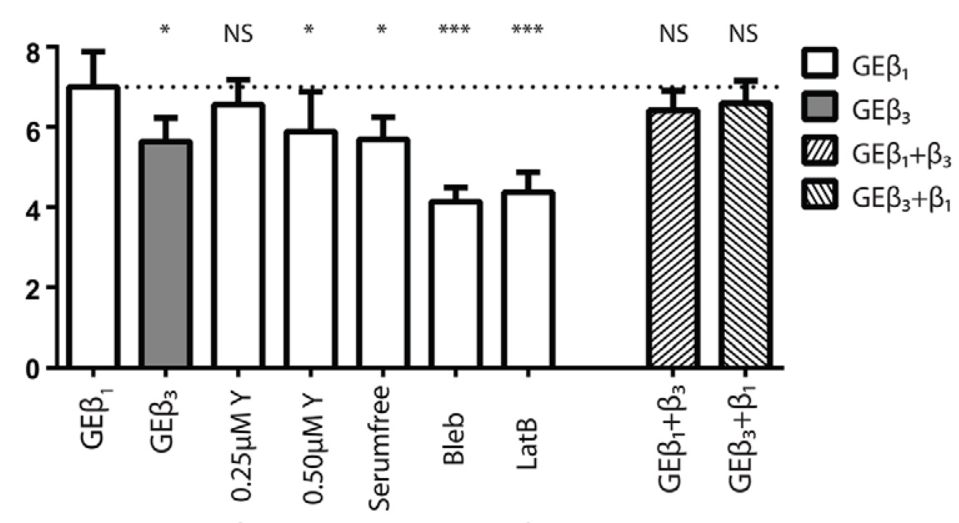

D

$\mathrm{GE} \beta_{1}$
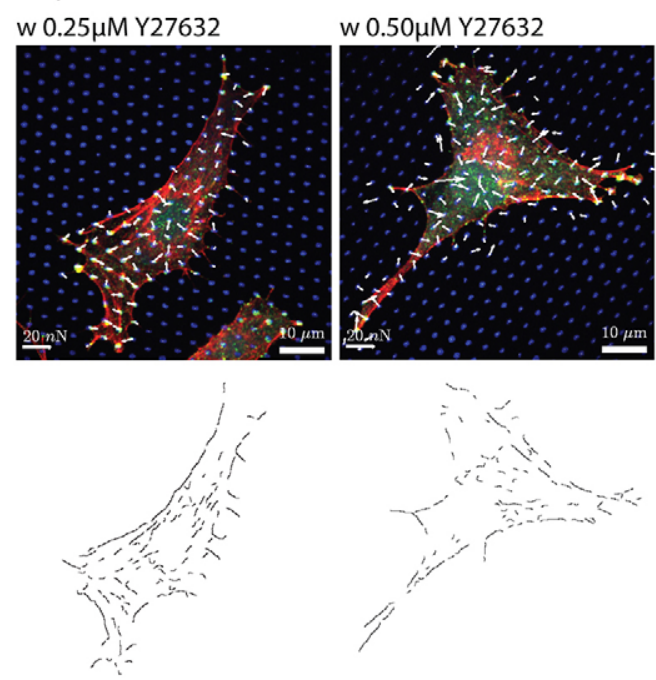

wo Serum
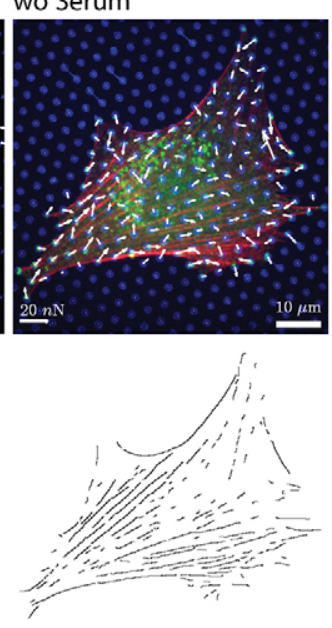

C

Fixed

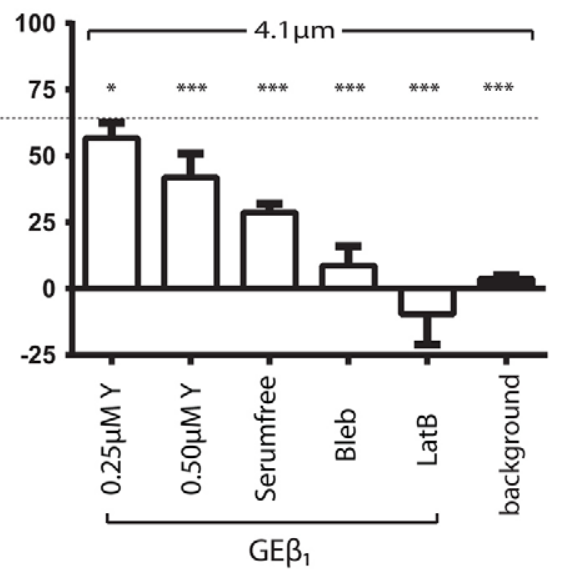

$\mathrm{GE} \beta_{1}$
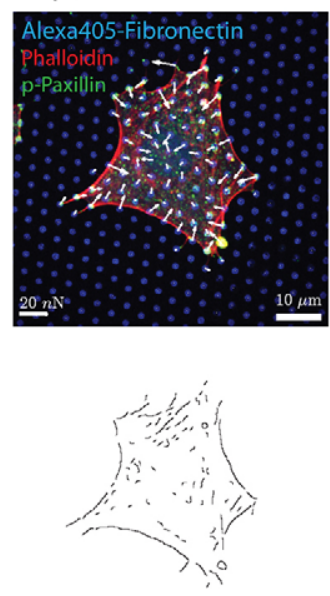

$\mathrm{GE} \beta_{3}$

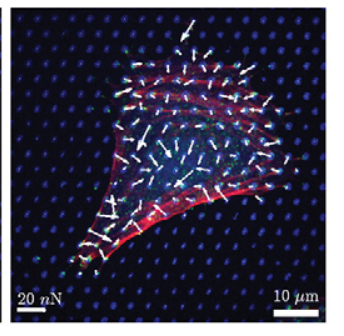

w $50 \mu \mathrm{M}$ Blebbistatin

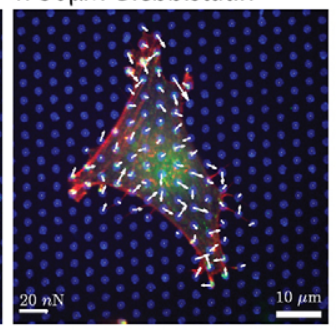

w $0.50 \mu \mathrm{M}$ Latrunculin B

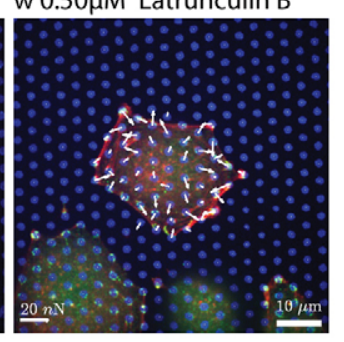

Fig. 5. See next page for legend.

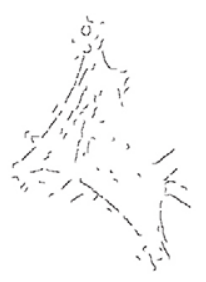


Fig. 5. Higher centripetal force fraction in cells using $\alpha 5 \beta 1$ correlates with longer cortical actin filaments that are dependent on ROCK- and myosin II activity. (A) Bar plots showing percentage centripetal force for the indicated cell lines on $6.9 \mu \mathrm{m}$ and $4.1 \mu \mathrm{m}$ PDMS pillars determined by live microscopy (left graph) or post-fixation analysis (middle and right graphs). Treatments in the right graph are $0.25 \mu \mathrm{M} Y$ and $0.5 \mu \mathrm{M}$ Y, Y27632 concentrations; bleb, $50 \mu \mathrm{M}$ blebbistatin; LatB, $0.5 \mu \mathrm{M}$ Latranculin $\mathrm{B}$. Background indicates forces measured in areas not covered by cells. (B) Bar plots of average cortical actin filament length of the indicated cell lines on $4.1 \mu \mathrm{m}$ PDMS pillars. Indicated treatments as in A, right graph. In all graphs, the mean $\pm 95 \%$ clearance level is shown and at least 15 cells were measured over three different experiments. Indicated $P$-values are compared to untreated $\beta 1$ expressing cells (marked by dotted lines); NS, $P>0.05$; ${ }^{*} P<0.05$; ${ }^{*} P<0.005$; ${ }^{* *} P<0.0005$ according to Mann-Whitney test. $(C, D)$ Representative images (top) and extracted actin cytoskeleton (bottom) for control (C) and treatment conditions (D). White arrows indicate magnitude and direction of forces measured. Scale bars: $20 \mathrm{nN}$ and $10 \mu \mathrm{m}$.

more randomly oriented. The ability of $\alpha 5 \beta 1$ to induce ROCKand myosin-II-mediated signaling, as demonstrated by us and others (Danen et al., 2002; Danen et al., 2005; Schiller et al., 2013; White et al., 2007), is important in this respect. We show that it allows cells to form long actin filaments that might support long-range force organization.

In conclusion, our findings show that both $\alpha 5 \beta 1$ and $\alpha v \beta 3$ integrins support force sensing and force generation, but $\alpha 5 \beta 1$ predominantly mediates centripetally oriented traction forces that are supported by ROCK- and myosin-II-mediated long actin filaments. By contrast, the shorter actin cables that are supported by $\alpha v \beta 3$ allow more random force application and might provide cells with increased actin cytoskeletal flexibility, allowing them to more dynamically respond to mechanical cues (Fig. 6). This might be particularly relevant in processes in which tissues go through extensive physical remodeling, such as embryonic development, angiogenesis and cancer progression where emergence of $\alpha v \beta 3$ has been documented.
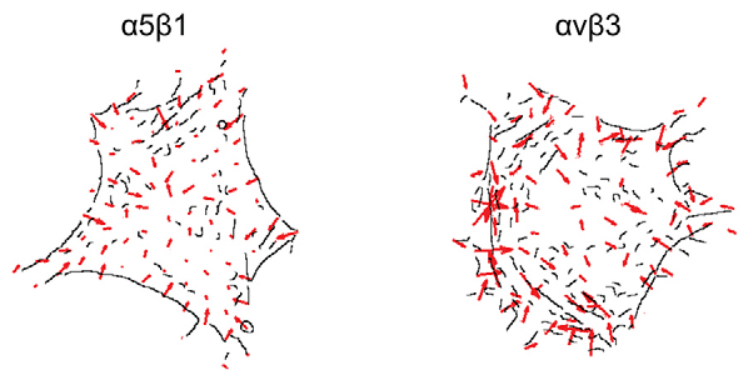

ROCK
long actin fibers
centripetal orientation of
traction forces

ROCK

short actin fibers

random orientation of traction forces increased flexibility/sensing

Fig. 6. Model for integrin regulated mechanotransduction. Both $\alpha 5 \beta 1$ and $\alpha v \beta 3$ integrins are able to support sensing and responding to mechanical cues from the environment (outside-in signaling) and to mediate force generation onto the ECM (inside-out signaling). ROCK- and myosinmediated long actin filaments are supported by $\alpha 5 \beta 1$ integrins and allow cells to apply centripetally oriented forces. Shorter actin filaments in $\alpha v \beta 3-$ expressing cells support more randomly oriented traction forces and might provide flexibility to reorganize the actin cytoskeleton in response to mechanical cues from the environment. Potential roles for alternative $\alpha \mathrm{v}$ integrins (e.g. $\alpha v \beta 1$ and $\alpha v \beta 6$ ) have not been tested here but might modulate the outcome of shifts in expression of $\alpha 5 \beta 1$ and $\alpha v \beta 3$.

\section{MATERIALS AND METHODS \\ FACS analysis}

For FACS, cells were detached using trypsin/EDTA and integrin surface expression levels were determined using primary antibodies (for human integrin $\beta 1$, AIIB2, Developmental Studies Hybridoma Bank, Iowa City, IA and for human integrin $\beta 3,23 C A$, Santa Cruz Biotechnology, Inc., Dallas, TX) and fluorescence-conjugated secondary antibodies (AlexaFluor-488-conjugated anti-rat-IgG or anti-rabbit-IgG, both from Invitrogen/Fisher Scientific, Breda, The Netherlands) and analyzing on a FACSCanto (Becton Dickinson, Breda, The Netherlands).

\section{Cell culture}

GD25 and GE11 cell lines expressing either $\alpha 5 \beta 1$ or $\alpha v \beta 3$ or both integrins were as described previously (Danen et al., 2002; Danen et al., 2005) and were selected for integrin expression using bulk FACS (supplementary material Fig. S1). Cells were cultured in Dulbecco's modified Eagle's medium (DMEM;, Invitrogen/Fisher Scientific) supplemented with $10 \%$ fetal bovine serum (HyClone, Etten-Leur, The Netherlands), $25 \mathrm{U} / \mathrm{ml}$ penicillin and $25 \mu \mathrm{g} / \mathrm{ml}$ streptomycin (Invitrogen/ Fisher Scientific cat. number 15070-063). For visualization of the actin cytoskeleton, cells were transduced using a lentiviral mCherry-LifeAct cDNA expression vector (provided by Olivier Pertz, University of Basel, Basel, Switzerland), selected in medium containing $2 \mu \mathrm{g} / \mathrm{ml}$ puromycin (Acros Organics/Fisher Scientific cat. number 227420500), and bulk sorted for mCherry expression using FACS (supplementary material Fig. S1C-F,J). MDA-MB-435s human breast cancer cells were cultured in RPMI medium 1640 (Invitrogen/Fisher Scientific) supplemented with $10 \%$ fetal bovine serum, $25 \mathrm{U} / \mathrm{ml}$ penicillin and $25 \mu \mathrm{g} / \mathrm{ml}$ streptomycin. NIH3T3 cells were culture in DMEM supplemented with $10 \%$ newborn calf serum, $25 \mathrm{U} / \mathrm{ml}$ penicillin and $25 \mu \mathrm{g} / \mathrm{ml}$ streptomycin.

\section{Cyclic cell stretching}

An in-house made, piezo-driven, uniaxial stretcher was used to apply cyclic stretch with defined frequency, duration and displacement, on cells adhered to a fibronectin-coated PDMS membrane. Membranes were generated by pipetting well mixed PDMS (Sylgard 184, Dow Corning, Midland, MI, USA) at a 1:10 (crosslinker:prepolymer) ratio inside a glass mold passivated with trichloro $(1 \mathrm{H}, 1 \mathrm{H}, 2 \mathrm{H}, 2 \mathrm{H}$-perfluorooctyl)silane (Sigma Aldrich, Zwijndrecht, The Netherlands) and incubating for 20 hours at $110^{\circ} \mathrm{C}$. This membrane was mounted on the stretcher, coated with $10 \mu \mathrm{g} / \mathrm{ml}$ fibronectin (Sigma Aldrich cat. number F1141) in phosphate buffered saline (PBS) and cells were seeded and incubated overnight in complete medium at $37^{\circ} \mathrm{C}$ and $5 \% \mathrm{CO}_{2}$ to allow full spreading. The stretcher was then mounted on a spinning-disk confocal microscope (see Materials and Methods, microscopy section), and was kept at $37^{\circ} \mathrm{C}$ by a stand-alone single loop temperature controller (\#3216, InvensysEuroterm, Alphen aan den Rijn, The Netherlands) connected to heaters and a thermo-coupler. LabVIEW (National Instruments, Austin, TX) scripts developed by Wim Pomp (Physics of Life Processes, Kamerlingh Onnes-Huygens Laboratory, Leiden University, Leiden, the Netherlands) and provided by the manufacturer of the controller unit (MCS-3D, SmarAct, Oldenburg, Germany) were used to drive two independent piezo motors (SLC2430s, SmarAct) that allowed uniaxial stretching. Images were collected before stretch application, after 2 hours of $10 \% 1 \mathrm{~Hz}$ stretching and after a subsequent 1 hour of $20 \% 1 \mathrm{~Hz}$ stretching.

\section{Characterization of stretcher strain field}

The strain field was quantified with help from Donato Civita (Physics of Life Processes, Kamerlingh Onnes-Huygens Laboratory, Leiden University, Leiden, The Netherlands) by stretching a membrane with a micro-contact printed hexagonal lattice of fluorescent dots (Alexa Fluor 647, Invitrogen). Two-dimensional image cross-correlation provided a deformation field over the entire substrate (supplementary material Fig. S2A). Differentiation using the Lagrangian strain tensor yielded the strain on every position on the substrate. The strain was $0.43 \%$ in the $x$ direction and $-0.18 \%$ in the $y$-direction for every $0.5 \%$ externally applied static strain. These results were homogeneous and reproducible over the entire substrate within a strain measurement error of $0.01 \%$. 
For characterizing strain at cyclic stretch conditions, a PDMS membrane with fluorescent beads dried on top was used and the piezo motors were run at $10 \%$ or $20 \%$ displacement at $0.01 \mathrm{~Hz}$ and a stack of images was obtained every 2 or 3 seconds, respectively to get the infocus image and calculate strain. A macroscopic strain of $10 \%$ and $20 \%$ resulted in $8.0 \%$ and $14.5 \%$ strain, respectively, on the central area of the membrane along the direction of global strain. The substrate showed $3.5 \%$ and $5.0 \%$ shrinkage, respectively, in the perpendicular direction (Fig. 1A; supplementary material Fig. S2A-D). Based on these measurements we calculated that the minimal strain was at $57^{\circ}$ and $60^{\circ}$, respectively relative to direction of macroscopic strain.

\section{PAA substrates}

PAA gels on 12-mm coverslips were made according to specifications adapted from Yeung et al. (Yeung et al., 2005). Briefly, autoclaved 12$\mathrm{mm}$ coverslips (Thermo Fisher cat. number 360302) were cleaned with $0.1 \mathrm{M} \mathrm{NaOH}$, and then rendered hydrophilic by incubating with $0.5 \% 3-$ aminopropyltrimethoxysilane (Sigma-Aldrich cat. number 281778). The coverslips were then washed thoroughly with sterile distilled water and incubated in $0.5 \%$ glutaraldehyde (Sigma-Aldrich cat. number G6257). Upon removal of the glutaraldehyde, the coverslips were left overnight to dry in a laminar flow cabinet. Coverslips of $10-\mathrm{mm}$ diameter (Thermo Fisher cat. number 360301) were rendered hydrophobic by incubating with a solution of $10 \%$ hydrocarbon-soluble siliconizing fluid (Surfa Sil; Thermo Fisher, cat. number TS-42800) in chloroform. Surfa Sil-treated coverslips were washed in $100 \%$ chloroform and then washed twice with methanol before being left overnight in a laminar flow cabinet to dry. PAA solutions were made with compositions of $7.5 \%$ acrylamide (Biorad cat. number 161-0141, Veenendaal, The Netherlands) and varying concentrations $(0.01 \%, 0.03 \%, 0.05,0.1 \%, 0.15 \%, 0.2 \%, 0.3 \%$ and $0.5 \%$ ) of bis-acrylamide (Biorad cat. number 161-0200) to a final volume of $1 \mathrm{ml}$. To this solution, $1.5 \mu \mathrm{l}$ TEMED (Thermo Fisher cat. number 17-1312-01) and $5 \mu \mathrm{l}$ of $10 \%$ ammonium persulfate were added to start polymerization. $10 \mu \mathrm{l}$ of this final solution was applied to the middle of each 12-mm coverslip. The $10-\mathrm{mm}$ coverslips were then placed on top of this solution to form a sandwich and left to polymerize for 30 minutes. $50 \mathrm{mM}$ HEPES was added and after 15 minutes the top coverslips were removed and the gels were washed once with $50 \mathrm{mM}$ HEPES. PAA gels were activated with an organic crosslinker by removing HEPES and submerging gels in a solution of $0.5 \mathrm{mM}$ sulfosyccinimidyl-6-(4'azido2 'nitrophenylamino)hexanoate (Thermo Fisher, cat. number 22589) and $50 \mathrm{mM}$ HEPES and placing under UV light (Philips HP3114, Eindhoven, The Netherlands). This step was repeated a second time after a wash with $50 \mathrm{mM}$ HEPES. The gels were then washed twice with $50 \mathrm{mM}$ HEPES and incubated overnight at $4^{\circ} \mathrm{C}$ in $10 \mu \mathrm{g} / \mathrm{ml}$ fibronectin (Sigma Aldrich cat. number F1141) in PBS. After washing with PBS, gels were allowed to equilibrate for 1 hour in complete culture medium at $37^{\circ} \mathrm{C}$ before seeding with 25,000 cells/well in complete medium. Cells were allowed to adhere and spread before fixation by incubating for two hours at $37^{\circ} \mathrm{C}$ and $5 \% \mathrm{CO}_{2}$.

\section{Analysis of stiffness of PAA gels by rheology}

Rheology experiments were performed with a stress-controlled rheometer (Physica MCR 501; Anton Paar, Graz, Austria) with assistance from Karin A. Jansen and Gijsje H. Koenderink (Biological Soft Matter Group, FOM Institute AMOLF, Amsterdam, The Netherlands) as previously described (Jansen et al., 2013). Briefly the PAA gel was polymerized at $21^{\circ} \mathrm{C}$ between a steel cone and plate $\left(40 \mathrm{~mm}\right.$ diameter, $\left.1^{\circ}\right)$ and shear storage modulus was recorded in real time during the polymerization $(\sim 1$ hour) by applying a small-amplitude oscillatory strain with amplitude $0.5 \%$ and frequency $3.14 \mathrm{rad} /$ second. After polymerization, PBS was added to the measuring chamber and the system was brought to $37^{\circ} \mathrm{C}$ while monitoring the shear storage modulus. The measured shear loss modulus was more than two orders of magnitude smaller than the storage component, hence was ignored.

\section{PAA and PDMS adhesion assay}

GE $\beta 1$ and GE $\beta 3$ cells were first incubated on ice with blocking antibodies targeting mouse integrin $\alpha 5$ (cat. number MAB1984,
Millipore, CA), mouse $\alpha \mathrm{v}$ (cat. number 552299 Becton Dickinson, Breda, The Netherlands), human $\beta 1$ (AIIB2) and human $\beta 3$ (23CA) for 30 minutes and then seeded on PAA gels (stiffness of $12.2 \mathrm{kPa}$ ) for 1 hour or on PDMS blocks (a 1:10 crosslinker to prepolymer ratio) for 30 minutes at $37^{\circ} \mathrm{C}$ and $5 \% \quad \mathrm{CO}_{2}$. They were then fixed with formaldehyde and cells on 6-10 different fields of view per condition were counted.

\section{Assays using PDMS micropillars}

Micropillars were used for cellular traction force measurements according to methodology described previously (Trichet et al., 2012; van Hoorn et al., 2014). A negative silicon wafer master was made using a two-step deep reactive ion etching (DRIE) process. Two different etching depths were obtained by subsequently applying two masks to the same wafer. A mask with $10 \times 10 \mathrm{~mm}$ arrays with circles of $2 \mu \mathrm{m}$ diameter and $4 \mu \mathrm{m}$ center-to-center distance in a hexagonal grid was used as a negative for the micropillar arrays and a mask with two rectangular spacers of $10 \times 2 \mathrm{~mm}$ was aligned on the sides of the arrays. The etching depth was varied for the micropillar arrays to make short and long pillars, calculated to have a bending stiffness of $66 \mathrm{nN} / \mu \mathrm{m}$ and $16 \mathrm{nN} / \mu \mathrm{m}$, respectively, using finite element modeling (van Hoorn et al., 2014). Using a published elastic model (Ghibaudo et al., 2008), we calculated that these bending stiffnesses corresponded to a Young's modulus in continuous (e.g. PAA) substrates of $\sim 47.2$ and $11.6 \mathrm{kPa}$; corresponding to a shear modulus of 15.7 and $3.87 \mathrm{kPa}$, respectively. The etching depth of the spacers was set to $50 \mu \mathrm{m}$, to enable high-resolution microscopy with inverted micropillar arrays (see Materials and Methods, microscopy section).

After passivation of the negative silicon master with trichloro silane (Sigma Aldrich), well-mixed PDMS at a 1:10 (crosslinker:prepolymer) ratio was poured over the wafer. After 20 hours at $110^{\circ} \mathrm{C}$, the PDMS was fully cured at a stiffness of $2.5 \mathrm{MPa}$ (as determined by tensile testing). The individual micropillar arrays were peeled off with two spacers on the sides. ECM stamping was performed using a flat piece of PDMS (1:30 ratio, cured 16 hours at $65^{\circ} \mathrm{C}$ ). Per stamp, a $40 \mu \mathrm{mix}$ of $50 \mu \mathrm{g} / \mathrm{ml}$ unlabeled fibronectin (Sigma Aldrich) and $10 \mu \mathrm{g} / \mathrm{ml}$ fibronectin conjugated to Alexa Fluor 405 or Alexa Fluor 647 (both from Invitrogen) was used. After stamping, the micropillars were blocked with $0.2 \%$ Pluronic (F-127, Sigma Aldrich) in PBS for 1 hour at room temperature and washed with PBS.

Cells were seeded in complete medium, serum-free medium or medium containing blocking antibodies targeting mouse integrin $\alpha \mathrm{v}$ subunit, and imaging of F-actin and labeled fibronectin was performed after cell spreading. For some analyses, after cell spreading, the medium was exchanged for medium containing 0.25 or $0.5 \mu \mathrm{M}$ Y27632 ROCK inhibitor (Tocris cat. number 1254, Bristol, UK); $50 \mu \mathrm{M}$ blebbistatin myosin II inhibitor (Calbiochem cat. number 203389, Merck KGaA, Darmstadt, Germany), or $0.5 \mu \mathrm{M}$ Latranculin B F-actin polymerization inhibitor (Calbiochem cat. number 428020), and further incubated for 1 hour followed by $4 \%$ formaldehyde fixation and immunostaining.

\section{Immunostaining}

Cells were fixed in $4 \%$ formaldehyde and then permeabilized with $0.1 \%$ Triton-X and $0.5 \%$ BSA in PBS. Immunostaining was performed for phosphorylated (pY188) paxillin (Biosource/Invitrogen cat. number 44$722 \mathrm{G}$; Becton Dickinson cat. number 610052) followed by secondary antibodies conjugated with Alexa488 (Invitrogen/Fisher Scientific cat. number A11008) or Alexa Fluor 647 (Jackson ImmunoResearch cat. number 115-605-006). Rhodamine-phalloidin (Sigma-Aldrich cat. \# 77418-1EA) or Alexa-Fluor-568-phalloidin (Fisher Emergo B.V. cat. number A12380, Thermo Fisher) was used to stain F-actin. Hoechst 33258 was used to visualize nuclei.

\section{Microscopy}

High-resolution imaging was performed on an in-house constructed setup based on an Axiovert200 microscope body (Zeiss, Sliedrecht, The Netherlands). Confocal imaging was achieved by means of a spinning disk unit (CSU-X1, Yokogawa, Amersfoort, The Netherlands). The

. 
confocal image was connected to an emCCD camera (iXon 897, Andor, Belfast, UK). IQ-software (Andor) was used for basic setup-control and data acquisition. Three laser lines were coupled through a polarizationmaintaining single-mode fiber, controlled using an Acousto-Optical Tunable Filter (AA Optoelectronics, Orsay, France): $405 \mathrm{~nm}$ (Crystalaser, Reno, NV), $488 \mathrm{~nm}$ (Coherent, Santa Clara, CA, USA) and $561 \mathrm{~nm}$ (Cobolt, Stockholm, Sweden). Incorporated $50 \mu \mathrm{m}$ spacers next to the micropillar arrays combined with a $100-\mu \mathrm{m}$ thick coverslip enabled the use of a high numerical aperture (1.4) objective with $100 \times$ magnification. For live-cell imaging and imaging of $3 \mathrm{~T} 3$ cells, a Nikon Eclipse Ti microscope in scanning confocal mode was used together with a $20 \times$ magnification $0.75 \mathrm{NA}$ dry air lens with internal $1.5 \times$ magnification and 4.184 scanner zoom to obtain a pixel size of $0.2 \mu \mathrm{m}$.

\section{Image analysis}

All image analysis was performed using specifically designed Matlab scripts (Mathworks, Natick, MA, USA). For cell area analysis, scripts generated by Hans de Bont (Division of Toxicology, Leiden Academic Center for Drug Research, Leiden, the Netherlands) were adapted to apply a rolling ball filter to the image followed by a median filter and subsequently cell detection and image segmentation was performed manually per image to best obtain area per single cell.

For cell-matrix adhesion analysis a cell mask was generated by passing the image of the actin channel through a Gaussian low pass filter. Subsequently, the background intensity was subtracted and the image was run through a sobel and a log-edge detection algorithm followed by image dilation and hole filling each time. The outputs were checked and new masks were generated manually as described above when the mask did not correctly correspond to the cell. Subsequently, for cell-matrix adhesion detection, the pY188 paxillin signal that was assigned to a cell within $20 \mu \mathrm{m}$ from the cell border was first passed through a Gaussian low pass filter, and signal that was four standard deviations larger than the average of the signal was assigned to cell-matrix adhesions. The binary adhesion images were then subjected to a hole-filling algorithm followed by watershed segmentation. The results were manually checked and images showing incorrect adhesion recognition due to low signal-tonoise ratio were excluded from analysis.

For the actin filament analysis, a rolling ball filter was applied to the actin signal inside the cell mask area. Then the signal one standard deviation above the mean was taken as foreground signal. To remove noise from the signal, the signal was shrunk, then singular pixels were removed and finally the image was dilated once. From this image, objects smaller than $0.2 \mu \mathrm{m}^{2}$ were removed and then the image was skeletonized, followed by connecting diagonals to connect neighboring filaments and then removing all branching points to analyze filaments separately. When analyzing cortical actin, only filaments within $2 \mu \mathrm{m}$ of the cell border were taken into account. For orientation analysis, all filaments were averaged over all cells and the output was convolved with a unit Gaussian to improve the visualization. This was then corrected for the square imaging window by calculating the maximum measurable fiber length in a given angle and weighing this correction per stretch condition by the percentage of a cell of measured average size falling outside of the imaging window if it were a circle (supplementary material Fig. S2E).

\section{Pillar deflection analysis}

Pillar deflections were determined with $\sim 30 \mathrm{~nm}$ precision using a specifically designed Matlab script (van Hoorn et al., 2014). Briefly, the exact pillar locations were determined from the labeled fibronectin fluorescence image using a fit to the cross-correlation function between a perfect binary circle and the local fluorescence of one pillar. The undeflected hexagonal grid was determined and used as reference to the determined pillar locations. The precision of the forces was dependent on the pillar bending stiffness, where the high- and low-stiffness pillars had a precision of $2 \mathrm{nN}$ and $0.5 \mathrm{nN}$, respectively.

Cell masks were generated using the same algorithm as for the cellmatrix adhesion analysis that was then dilated. The pillars under this dilated image that had a deflection larger than $0.06 \mu \mathrm{m}$ in the fixed and integrin blocking assay and larger than $0.2 \mu \mathrm{m}$ for all other live assays were taken for analysis. Total force was calculated by adding all the absolute deflections and multiplying it by the bending stiffness. The centripetal force percentage was obtained by dividing the radial components of the forces (the forces that point towards the center of the generated cell mask) by the total cellular force.

\section{Statistical analysis}

To calculate significance between two conditions, the Mann-Whitney U test was used. To quantify the PAA substrate responses, a cumulative Gaussian distribution was fitted to the data and the half response point (the mean of the distribution) was compared between the conditions using the F-test in the GraphPad Prism 6 program (GraphPad Software, La Jolla, CA).

\section{Acknowledgements}

We thank O. Pertz for providing the mCherry-LifeAct construct; W. Pomp and D. Civita for assistance in setting up cyclic stretcher; K. A. Jansen and G. H. Koenderink for their help in characterizing PAA gels; and $\mathrm{H}$. De Bont for assistance with image analysis.

\section{Competing interests}

The authors declare no competing or financial interests.

\section{Author contributions}

H.E.B. performed the experiments. H.E.B., H.v.H., D.M.D., T.S., and E.H.J.D. designed the experiments and analyzed and interpreted data. H.E.B. and E.H.J.D wrote the manuscript.

\section{Funding}

Support for this work came from the Netherlands Organization for Scientific Research NWO-FOM [grant numbers $09 M M C 03$ to H.E.B. and 09MMC01 to H.v.H. and D.M.D.]; and from the Dutch Cancer Society [grant number UL 2010-4670].

\section{Supplementary material}

Supplementary material available online at

http://jcs.biologists.org/lookup/suppl/doi:10.1242/jcs.156950/-/DC1

\section{References}

Bouvard, D., Pouwels, J., De Franceschi, N. and Ivaska, J. (2013). Integrin inactivators: balancing cellular functions in vitro and in vivo. Nat. Rev. Mol. Cell Biol. 14, 430-442.

Choquet, D., Felsenfeld, D. P. and Sheetz, M. P. (1997). Extracellular matrix rigidity causes strengthening of integrin-cytoskeleton linkages. Cell 88, 3948.

Danen, E. H. J., Sonneveld, P., Brakebusch, C., Fassler, R. and Sonnenberg, A. (2002). The fibronectin-binding integrins alpha5beta1 and alphavbeta3 differentially modulate RhoA-GTP loading, organization of cell matrix adhesions, and fibronectin fibrillogenesis. J. Cell Biol. 159, 1071-1086.

Danen, E. H. J., van Rheenen, J., Franken, W., Huveneers, S., Sonneveld, P., Jalink, K. and Sonnenberg, A. (2005). Integrins control motile strategy through a Rho-cofilin pathway. J. Cell Biol. 169, 515-526.

Desgrosellier, J. S. and Cheresh, D. A. (2010). Integrins in cancer: biological implications and therapeutic opportunities. Nat. Rev. Cancer 10, 9-22.

Friedland, J. C., Lee, M. H. and Boettiger, D. (2009). Mechanically activated integrin switch controls alpha5beta1 function. Science 323, 642-644.

Ghibaudo, M., Saez, A., Trichet, L., Xayaphoummine, A., Browaeys, J., Silberzan, P., Buguin, A. and Ladoux, B. (2008). Traction forces and rigidity sensing regulate cell functions. Soft Matter 4, 1836-1843.

Guilluy, C., Swaminathan, V., Garcia-Mata, R., O'Brien, E. T., Superfine, R. and Burridge, K. (2011). The Rho GEFs LARG and GEF-H1 regulate the mechanical response to force on integrins. Nat. Cell Biol. 13, 722-727.

Hoffman, B. D., Grashoff, C. and Schwartz, M. A. (2011). Dynamic molecular processes mediate cellular mechanotransduction. Nature 475, 316-323.

Huveneers, S., Truong, H., Fässler, R., Sonnenberg, A. and Danen, E. H. J. (2008). Binding of soluble fibronectin to integrin alpha5 beta1 - link to focal adhesion redistribution and contractile shape. J. Cell Sci. 121, 2452-2462.

Hynes, R. O. (2002). Integrins: bidirectional, allosteric signaling machines. Cell 110, 673-687.

Jansen, K. A., Bacabac, R. G., Piechocka, I. K. and Koenderink, G. H. (2013). Cells actively stiffen fibrin networks by generating contractile stress. Biophys. J. 105, 2240-2251.

Katsumi, A., Naoe, T., Matsushita, T., Kaibuchi, K. and Schwartz, M. A. (2005). Integrin activation and matrix binding mediate cellular responses to mechanical stretch. J. Biol. Chem. 280, 16546-16549.

Lin, G. L., Cohen, D. M., Desai, R. A., Breckenridge, M. T., Gao, L., Humphries, M. J. and Chen, C. S. (2013). Activation of beta 1 but not beta 3 integrin increases cell traction forces. FEBS Lett. 587, 763-769. 
Miao, H., Li, S., Hu, Y.-L., Yuan, S., Zhao, Y., Chen, B. P. C., PuzonMcLaughlin, W., Tarui, T., Shyy, J. Y.-J., Takada, Y. et al. (2002). Differential regulation of Rho GTPases by $\beta 1$ and $\beta 3$ integrins: the role of an extracellular domain of integrin in intracellular signaling. J. Cell Sci. 115, 2199-2206.

Mills, G. B. and Moolenaar, W. H. (2003). The emerging role of lysophosphatidic acid in cancer. Nat. Rev. Cancer 3, 582-591.

Roca-Cusachs, P., Gauthier, N. C., Del Rio, A. and Sheetz, M. P. (2009). Clustering of alpha(5)beta(1) integrins determines adhesion strength whereas alpha(v)beta(3) and talin enable mechanotransduction. Proc. Natl. Acad. Sci. USA 106, 16245-16250.

Roca-Cusachs, P., Iskratsch, T. and Sheetz, M. P. (2012). Finding the weakest link: exploring integrin-mediated mechanical molecular pathways. J. Cell Sci. 125, 3025-3038.

Schiller, H. B. and Fässler, R. (2013). Mechanosensitivity and compositional dynamics of cell-matrix adhesions. EMBO Rep. 14, 509-519.

Schiller, H. B., Hermann, M.-R., Polleux, J., Vignaud, T., Zanivan, S., Friedel, C. C., Sun, Z., Raducanu, A., Gottschalk, K.-E., Théry, M. et al. (2013). $\beta 1$ - and $\alpha$ V-class integrins cooperate to regulate myosin II during rigidity sensing of fibronectin-based microenvironments. Nat. Cell Biol. 15, 625636.
Trichet, L., Le Digabel, J., Hawkins, R. J., Vedula, S. R. K., Gupta, M., Ribrault, C., Hersen, P., Voituriez, R. and Ladoux, B. (2012). Evidence of a large-scale mechanosensing mechanism for cellular adaptation to substrate stiffness. Proc. Natl. Acad. Sci. 109, 6933-6938.

van Hoorn, H., Harkes, R., Spiesz, E. M., Storm, C., van Noort, D., Ladoux, B. and Schmidt, T. (2014). The nanoscale architecture of force-bearing focal adhesions. Nano Lett. 14, 4257-4262.

White, D. P., Caswell, P. T. and Norman, J. C. (2007). $\alpha \vee \beta 3$ and $\alpha 5 \beta 1$ integrin recycling pathways dictate downstream Rho kinase signaling to regulate persistent cell migration. J. Cell Biol. 177, 515-525.

Wolfenson, H., Lavelin, I. and Geiger, B. (2013). Dynamic regulation of the structure and functions of integrin adhesions. Dev. Cell 24, 447-458.

Woods, D., Cherwinski, H., Venetsanakos, E., Bhat, A., Gysin, S., Humbert, M., Bray, P. F., Saylor, V. L. and McMahon, M. (2001). Induction of $\beta 3$-integrin gene expression by sustained activation of the Ras-regulated Raf-MEK-extracellular signal-regulated kinase signaling pathway. Mol. Cell. Biol. 21, 3192-3205.

Yeung, T., Georges, P. C., Flanagan, L. A., Marg, B., Ortiz, M., Funaki, M., Zahir, N., Ming, W., Weaver, V. and Janmey, P. A. (2005). Effects of substrate stiffness on cell morphology, cytoskeletal structure, and adhesion. Cell Motil. Cytoskeleton 60, 24-34. 